\title{
A Case Report of Etiology of Cerebral Venous Sinus Thrombosis Developed After Spinal Anesthesia in Asteroid, Doping Using Young Athlete
}

\author{
Aylin Bican Demir ${ }^{\mathrm{a}, \mathrm{c}}$, Gulfer Atasayar ${ }^{\mathrm{a}}$, Necdet Karlia, \\ Ozlem Taskapilioglu ${ }^{\mathrm{a}}$, Ferda Kahveci ${ }^{\mathrm{b}}$
}

\begin{abstract}
Cerebral venous thrombosis (SVT) was defined for the first time in 1875. It is a rare entity that can occur with different clinics. Superior sagittal sinus is affected most $(70-80 \%)$, then transverse, sigmoid, and in a lesser extent, cavernous sinus involvement is seen. Cortical and cerebellar veins may also be involved. A 22-year-old male patient presented to the emergency room with mental confusion and seizures. According to the information received from his relatives, he was actively involved in sports for 2 years. That he was using amino acid weighted and high-energy multi-vitamin complexes within a day, and monthly having an injection called Deca-Durabolin: (nandrolone decanoate), is learned. One week before his coming to the hospital, that he had cruciate ligament surgery on his left knee and that spinal anesthesia was administered to him during the operation is determined. Thereafter, it is learnt that he had generalized tonic-clonic seizure and impairment of consciousness was added when he came to the hospital. During the patient's neurologic examination in the emergency service, it is observed that his consciousness tended to fall asleep, and existing of bilateral papilledema and spontaneous movements in the right, and bilateral Babinski was positive. In brain computed tomography and magnetic resonance imaging (MRI) of the patient, in the left temporo-parieto-occipital hemorrhagic infarct and in venous MR angiography, in left transfer sinus, sigmoid sinus and jugular sinus complete thrombus, in right transfer and lateral sinus, reduction in blood stream and a view matched with thrombus were detected. On the 16th day of his hospitalization, brain death was determined in patient. At the arrival of the patient to the clinic, mental confusion, being male, to be accompanied by intracranial hemorrhage shows that poor prognosis.
\end{abstract}

Manuscript accepted for publication February 5, 2014

aDepartment of Neurology, Faculty of Medicine, Uludag University, Bursa, Turkey

${ }^{\mathrm{b}}$ Department of Intensive Care, Faculty of Medicine, Uludag University, Bursa, Turkey

${ }^{\mathrm{c}}$ Corresponding author: Aylin Bican Demir, Department of Neurology,

Faculty of Medicine, Uludag University, 16059, Gorukle, Bursa, Turkey.

Email: aylinbican@mynet.com

doi: http://dx.doi.org/10.14740/jnr263w
Keywords: Cerebral venous sinus thrombosis; Spinal anesthesia; Steroid; Doping; Young athlete; Epilepsy

\section{Introduction}

Cerebral venous thrombosis (SVT) was defined for the first time in 1875 . It is a rare entity that can occur with different clinics. Cerebral venous sinus thrombosis associated secondary strokes are rarer than arterial stroke and usually seen in young people. It is responsible for $1-2 \%$ of all stocks in adults [1].

In the etiology pregnancy, puerperium, use of oral contraceptives (OC), coagulation disorders, intracranial infections, cranial tumors, penetrating head traumas, lumbar puncture, malignancy, dehydration, inflammatory bowel disease, connective tissue diseases, Behcet's disease, sarcoidosis, nephrotic syndrome, parenteral infusions and various medicines exist. Despite all research, in $20-25 \%$ of patients, the cause cannot be determined $[2,3]$.

Superior sagittal sinus is affected most $(70-80 \%)$, then transverse, sigmoid, and in a lesser extent, cavernous sinus involvement is seen. Cortical and cerebellar veins may also be involved. In one-third of the cases, more than one sinus are affected [4].

Clinical occurs depending on the place and the formation speed of thrombus. The most common symptom is headache (74-90\%). Other symptoms are seizures, focal neurological deficits (hemiparesis/hemihypoesthesia), papilledema, isolated intracranial hypertension (headache, papilledema, visual loss, nausea, dizziness and drowsiness), aphasia and hemianopsia. In treatment, oral antithrombotic drugs, low molecular weight heparin, intravenous thrombolysis, local thrombolysis, or a combination thereof are used [1].

\section{Case Report}

A 22-year-old male patient presented to the emergency room with mental confusion and seizures. According to the information received from his relatives, he was actively involved 

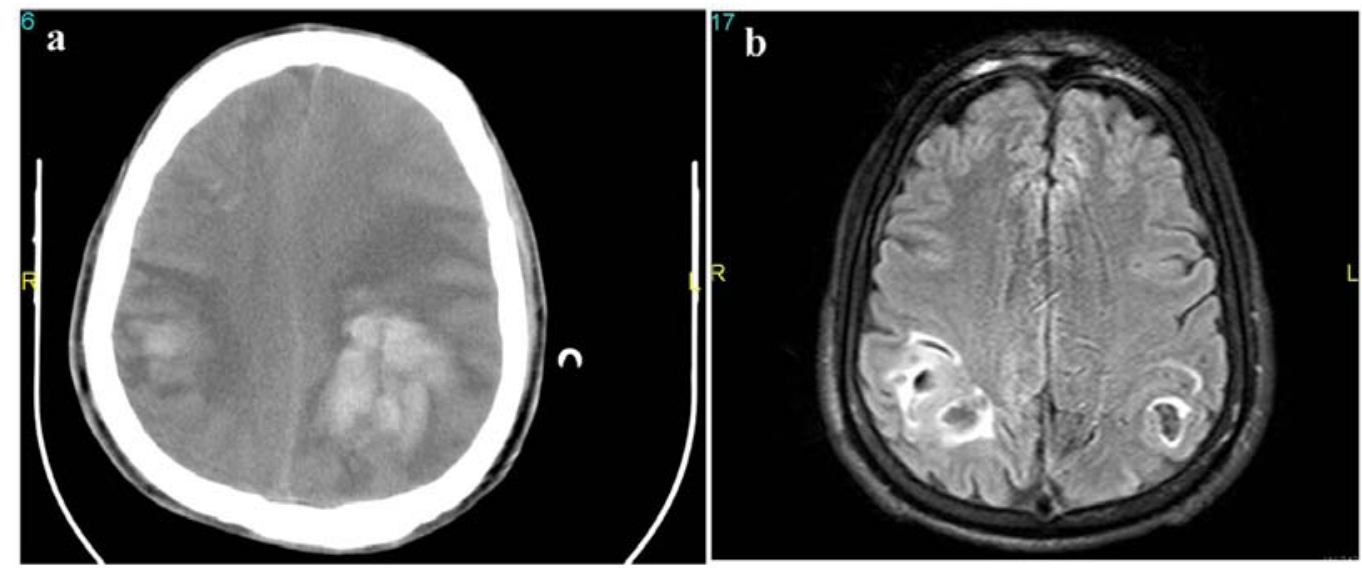

Figure 1. (a) Brain computed tomography of the patient, in the left temporo-parieto-occipital hemorrhagic infarct. (b) Magnetic resonance imaging (MRI) of the patient, in the left temporo-parieto-occipital hemorrhagic infarct.

in sports for 2 years. That he was using amino acid weighted and high-energy multi-vitamin complexes within a day, and monthly having an injection called Deca-Durabolin: (nandrolone decanoate), is learned. One week before his coming to the hospital, that he had cruciate ligament surgery on his left knee and that spinal anesthesia was administered to him during the operation is determined. In spite of not having any complaints soon after the operation, after 7 days, the patient developed a sudden headache. Thereafter, it is learnt that he had generalized tonic-clonic seizure and impairment of consciousness was added when he came to the hospital. During the patient's neurologic examination in the emergency service, it is observed that his consciousness tended to fall asleep, and existing of bilateral papilledema and spontaneous movements in the right, and bilateral Babinski was positive. In brain computed tomography $(\mathrm{CT})$ and magnetic resonance imaging (MRI) of the patient, in the left temporo-parietooccipital hemorrhagic infarct (Fig. 1a, b) and in venous MR

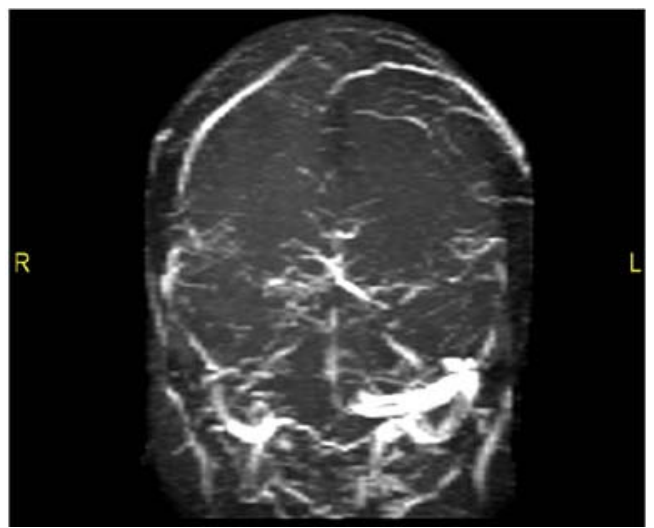

Figure 2. Venous MR angiography, in left transfer sinus, sigmoid sinus and jugular sinus complete thrombus, in right transfer and lateral sinus, reduction in blood stream and a view matched with thrombus were detected. angiography, in left transfer sinus, sigmoid sinus and jugular sinus complete thrombus, in right transfer and lateral sinus, reduction in blood stream and a view matched with thrombus (Fig. 2) were detected. To the patient, for 1 day intravenous heparin and for 2 days low-molecular-weight heparin were administered. Mannitol was injected as anti-edema. Focal motor seizures appeared on the right arm and leg in due course of his follow-up in intensive care. Status epilepticus developed later. In EEG, theta activity like significant sharp wave was observed in the right santro-parieto-occipital (Fig. $3)$. In status protocol intravenous levetiracetam and phenytoin were administered to the patient. After $24 \mathrm{~h}$, the patient's seizures were taken under control. Upon that hemorrhagic areas increased and the patient's anisocoria occurred, neurosurgery consulted in terms of intracranial pressure and because bleeding parameters were high, surgery was not considered. As clinically and radiologically diagnosed STV patients were examined, he was run all laboratory routine workups such as chest X-ray, electrocardiogram, hemogram, renal function tests, liver function tests, blood electrolytes and sedimentation rate. Vasculitis markers, thyroid function tests, protein $\mathrm{C}$ and protein $\mathrm{S}$, antithrombin III levels, anticardiolipin antibodies, lupus anticoagulant and homocysteine levels were examined and normally concluded. It drew attention that patient's creatine kinase level was 3,000 when he first came to the hospital and it spontaneously decreased to the level of 320s during the follow-up. That rise and spontaneous decrease was considered as a result of active sports done by patient. On the 10th day of the follow-up, filter was placed into vena kava inferior. The patient's situation was consulted to hematology unit; they could not find any primer hematological subject which may cause hypercoagulopathy and it was thought that patient's use of anabolic steroid, doping-purpose multivitamin and proteic substances (L-arginine, A, E, vitamin D, carnitine) and spinal anesthesia that he has undergone recently caused hypercoagulopathy. On the 


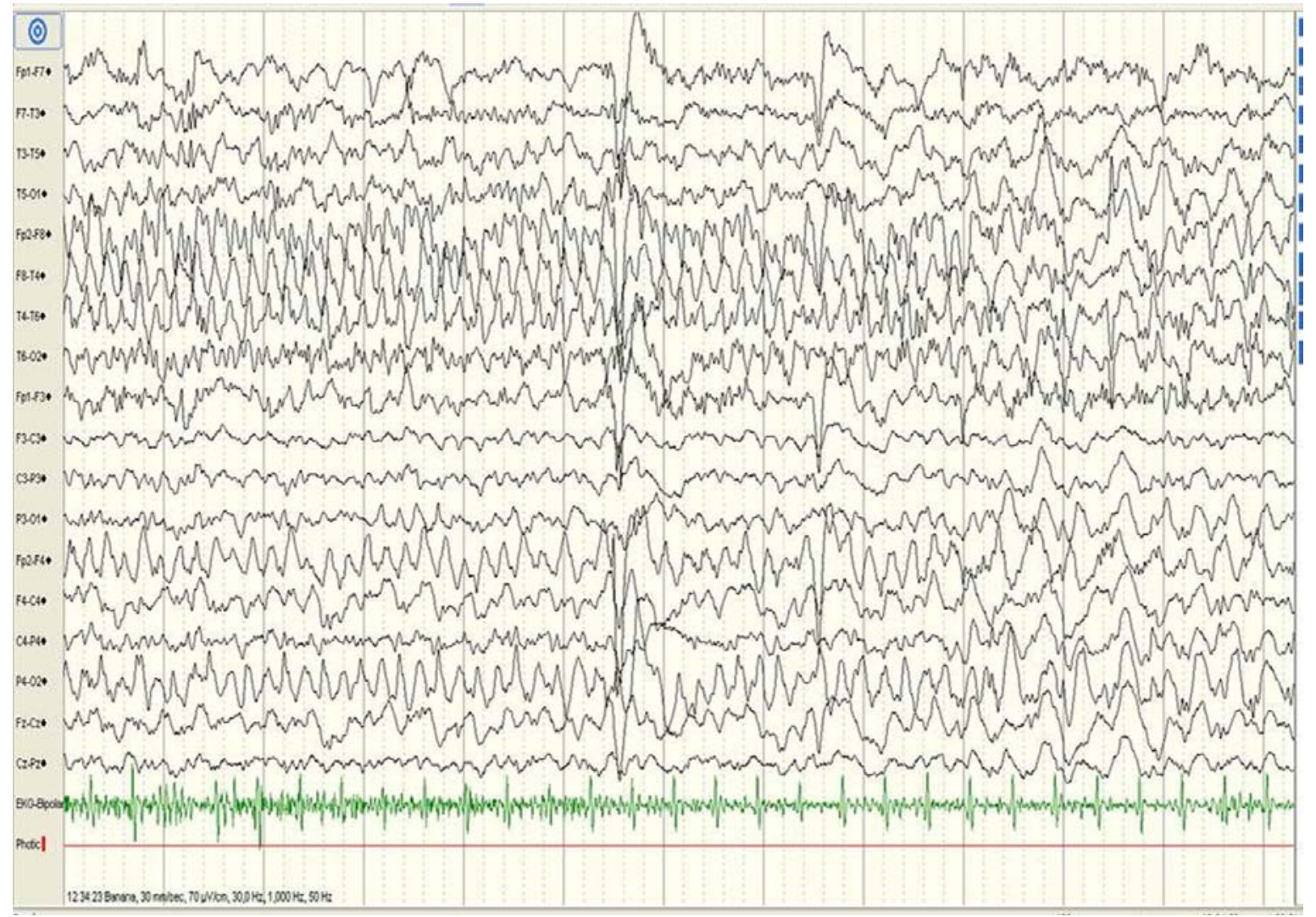

Figure 3. In EEG, theta activity like significant sharp wave was observed in the right santro-parieto-occipital.

16th day of his hospitalization, brain death was determined in patient.

\section{Discussion}

SVT can clinically mimic different kinds of neurological disorders including ischemic or hemorrhagic stroke, brain abscess, tumor, encephalopathy, idiopathic intracranial hypertension and migraine. Notwithstanding that it is usually seen in young patients, cerebral venous sinus thrombosis can be seen in all age groups [1]. Our case was a 22-year-old male patient. In our case, at first headaches and bilateral papilledema existed, generalized tonic-clonic seizures occurred, and after impairment of consciousness predominated. Initial and subsequent symptoms of our patient were consistent with cerebral venous sinus thrombosis clinic.

The radiological examinations for the diagnosis of SVT are brain MRI and MR venography. In case of doubt and only in the event of cricked cortical veins, the cerebral in which venous phase is considered DSA may be required. Brain CT is pathological in only $10-20 \%$ of the cases [4-7]. In our case, in his cranial CT hemorrhagic infarction and in venous MR angiography, there were symptoms regarding to venous sinus thrombosis.
In the literature, Terazzi et al's study, it is found that in etiology OC use is the most common, and the rate is determined as $47 \%$. In the studies, that inmost of the female patients in etiology OC use is seen and in addition, that one or more of the other risk factors existed is determined [8].

Sahraian et al discussed in literature a case of a 22-yearold sportsman who has androgenic injection monthly. The patient who presented with headache, nausea and papilledema was treated for cerebral venous sinus thrombosis with heparin. They mentioned that patient increased coagulation factors or platelet activation and so he could cause arterial and venous thrombosis [9]. Jaillard et al mentioned a case of venous sinus thrombosis that developed in again a 32-yearold young sportsman after androgen use [10]. Like similar cases in the literature, in our case, the patient was a 22-yearold sportsman and it was learnt that he used instensely amino acid weighted and high-energy multi-vitamin complexes and had injection of androgen. After that, the case was diagnosed as venous infarction with increasing hemorrhagic areas which are opened to the cerebral ventricles.

According to a 29-case study of the literature, headache in patients with venous sinus thrombosis and clinical emerged after lumbar puncture cases. Here, it is mentioned that after the lumbar puncture, spinal dural injury developed and as a result of intracranial CSF hypotension, it ended up 
with acute dilatation of cerebral vessels with orthostatic headache and venous stasis [11]. Oz et al presented a case of a young man that ended up with clinically headache, radiologically venous sinus thrombosis and subarachnoid hemorrhage after spinal anesthesia applied during a knee surgery [12]. In our case, our attention has been drawn to the fact that a week prior to our patient's admission to the hospital, he had a cruciate ligament surgery on his left knee and during the process was under spinal anesthesia.

While arterial thrombus is critical for the platelet activation, coagulation mechanisms of venous thrombus are important. Therefore, anticoagulants are primarily used in the treatment of SVT. Unfractionated heparin is a safe and effective treatment for SVT. The use of anticoagulants is controversial because of the risk of hemorrhagic infarctions bleeding. However, despite the fact that risk of intracranial hemorrhage increases and there is controversy about its indications, heparin as anticoagulant therapy is the first choice even in the presence of intracranial hemorrhage. If the patient deteriorates despite adequate heparinization, thrombolytic therapy can be given with selective catheterization [1]. The cases in the literature were non-complex cases of infarction secondary to thrombosis and were more appropriate in terms of heparinization.

In our case, we treated him with intravenous heparin and then followed after with low-molecular-weight heparin. Risk factors that exacerbate prognosis are male gender, advanced age, coma situation, accompanied by mental disorders, intracranial bleeding initially, keeping the deep cortical veins, FAQ infection and presence of cancer [13].

SVT may occur in a wide variety of neurological tables. The possibility of a missed diagnosis in patients without focal neurological signs is high. The patients who are beginner or have changing character headache and focal neurologic findings should be considered in the diagnosis of SVT and immediately radiological investigations should be done and treatment should be initiated.

As in our case, the patient's long time use of androgen, multi-vitamin formed venous infarct predisposition, the application of spinal anesthesia created a stage/environment prone to cerebral venous stanza resulting in hemorrhage venous infarct. At the arrival of the patient to the clinic, mental confusion, being male, to be accompanied by intracranial hemorrhage shows that poor prognosis.

\section{Conflict of Interest}

The authors state no conflict of interest.

\section{References}

1. Einhaupl K, Bousser MG, de Bruijn SF, Ferro JM, Martinelli I, Masuhr F, Stam J. EFNS guideline on the treatment of cerebral venous and sinus thrombosis. Eur J Neurol. 2006;13(6):553-559.

2. Ferro JM, Bacelar-Nicolau H, Rodrigues T, BacelarNicolau L, Canhao P, Crassard I, Bousser MG, et al. Risk score to predict the outcome of patients with cerebral vein and dural sinus thrombosis. Cerebrovasc Dis. 2009;28(1):39-44.

3. Ferro JM, Canhao P, Bousser MG, Stam J, Barinagarrementeria F. Cerebral vein and dural sinus thrombosis in elderly patients. Stroke. 2005;36(9):1927-1932.

4. Damak M, Crassard I, Wolff V, Bousser MG. Isolated lateral sinus thrombosis: a series of 62 patients. Stroke. 2009;40(2):476-481.

5. Mehndiratta MM, Garg S, Gurnani M. Cerebral venous thrombosis--clinical presentations. J Pak Med Assoc. 2006;56(11):513-516.

6. Alberti A, Venti M, Biagini S. Headache and cerebral vein and sinus thrombosis. Front Neurol Neurosci. 2008;23:89-95.

7. Gerber S, Rodallec M, Botto E, Espinoza S, Mellerio C, Zuber M, Zins M. Fatal acute hemorrhagic venous infarction due to the thrombosis of the draining vein of a developmental venous abnormality. J Neuroradiol. 2010;37(4):252-254.

8. Terazzi E, Mittino D, Ruda R, Cerrato P, Monaco F, Sciolla R, Grasso E, et al. Cerebral venous thrombosis: a retrospective multicentre study of 48 patients. Neurol Sci. 2005;25(6):311-315.

9. Sahraian MA, Mottamedi M, Azimi AR, Moghimi B. Androgen-induced cerebral venous sinus thrombosis in a young body builder: case report. BMC Neurol. 2004;4(1):22.

10. Jaillard AS, Hommel M, Mallaret M. Venous sinus thrombosis associated with androgens in a healthy young man. Stroke. 1994;25(1):212-213.

11. Miglis MG, Levine DN. Intracranial venous thrombosis after placement of a lumbar drain. Neurocrit Care. 2010;12(1):83-87.

12. Oz O, Akgun H, Yucel M, Battal B, Ipekdal HI, Ulas $\mathrm{UH}$, Demirkaya $\mathrm{S}$, et al. Cerebral venous thrombosis presenting with subarachnoid hemorrhage after spinal anesthesia. Acta Neurol Belg. 2011;111(3):237-240.

13. Bentley JN, Figueroa RE, Vender JR. From presentation to follow-up: diagnosis and treatment of cerebral venous thrombosis. Neurosurg Focus. 2009;27(5):E4. 